\title{
A Simplified Approach for Quasi-linear Power Amplifier Distortion Evaluation
}

\author{
Alberto Santarelli ${ }^{1}$, Rudi Paolo Paganelli ${ }^{2}$, Alberto Costantini ${ }^{1}$, Giorgio Vannini ${ }^{3}$, Fabio Filicori ${ }^{1}$ \\ ${ }^{1}$ University of Bologna, Department of Electronics, Viale Risorgimento 2, 40136 Bologna, Italy, +390512093039 \\ ${ }^{2}$ IEIIT-CNR, Department of Bologna, Viale Risorgimento 2, 40136 Bologna, Italy, +390512093845 \\ ${ }^{3}$ University of Ferrara, Department of Engineering, Via Saragat 1, 44100 Ferrara, Italy, +390532974884
}

\begin{abstract}
The paper presents a simplified approach for the evaluation of mild distortion in highly linear power amplifiers (PA) for microwave communications. In particular, it is shown how intermodulation distortion (IMD) can be accurately predicted on the basis of a single-tone power-swept Harmonic Balance analysis instead of using two-tone or multi-tone analyses leading to time-consuming and computationally expensive iterative PA design procedures. Moreover, simple equations provided in the paper show that common design specifications given in terms of maximum acceptable IMD are conveniently converted into constraints on a suitable non linearity index, involving both $\mathrm{AM} / \mathrm{AM}$ and $\mathrm{AM} / \mathrm{PM}$ amplifier characteristics. Experimental validation is provided in the paper on the basis of a $50 \Omega$-loaded GaAs $600 \mu \mathrm{m}-\mathrm{PHEMT}$ based power amplifier simulated with Agilent ADS.
\end{abstract}

\section{INTRODUCTION}

In recent years, an increasing demand for high-linearity amplifiers has been observed in broadband digital radio systems [3]-[8]. In particular, spectrally-efficient modulations, needed to face strong limitations in frequency band resources, are usually non-constant envelope schemes requiring challenging linearity constraints from RF power amplifier final stages. In such a context, power amplifier mild non-linearity evaluation tools become a very important issue in order to meet specifications during the design phase. Traditional and simple tests, such as harmonic distortion and scalar gain compression, do not seem to be valuable tools when dealing with highly-linear power amplifier for communication systems, since they involve a non realistic input test signal (constant amplitude carrier, no modulation, zero bandwidth) and only out-of-band harmonics at the output port. Instead, two-tone intermodulation (IM) analysis seems a much more meaningful test in this context. In fact, it involves an input test signal with non-zero bandwidth (amplitude and phase modulation) and in-band distortion products at the output. More recently, even more realistic tests, in the frame of a digitally modulated communication scheme, are considered such as the evaluation of the Adjacent and the Co-Channel Power Ratio [6],[8]. The simulation of all of these non-linearity tests (IMR, ACPR, CCPR,...) usually involves both a very accurate model, taking into account the non-linear dynamic behavior of the power amplifier/electron device, and very sophisticated numerical simulation tools in the framework of Computer
Aided Design environments (multi-tone HB, envelope algorithms, etc.) .

In the Section II of the paper, it is shown how meaningful IMD evaluation tests, such as third- and fifthorder IMR, can be accurately evaluated on the basis of a simple power swept single-tone $\mathrm{HB}$ analysis, provided that an accurate non-linear dynamic model of the involved electron device is available. Since standard PA design procedures usually involve iterative evaluation of the circuit performance under large signal operation, this turns out to be a noticeable advantage, leading to design processes much more numerically efficient and less time consuming. Moreover, in the Section III it is shown how the design specification on maximum acceptable IMD can be conveniently converted into an equivalent specification on a suitably defined non-linearity index involving both $\mathrm{AM} / \mathrm{AM}$ and $\mathrm{AM} / \mathrm{PM}$ amplifier characteristics. Experimental validation of the theoretical developments is eventually provided in Section IV.

\section{HIGHLY LiNEAR PA DistorTION EVALUATION}

Power amplifier intermodulation (IM) distortion prediction is considered here. To this aim a two-tone sinusoidal excitation is assumed at frequencies: $\omega_{1}=\omega_{0}-\Delta \omega / 2, \quad \omega_{2}=\omega_{0}+\Delta \omega / 2$, such that: $\Delta \omega<<\omega_{0}$. The instantaneous incident wave $a_{i n}(t)$ at the input port of the power amplifier (scalar quantity, $50 \Omega$ normalization) can be conveniently expressed as:

$$
a_{\text {in }}(t)=\operatorname{Re}\left\{\bar{a}(t) \cdot e^{j \omega_{0} t}\right\}
$$

where:

$$
\bar{a}(t)=\bar{a}_{1} e^{j\left(\omega_{1}-\omega_{0}\right) t}+\bar{a}_{2} e^{j\left(\omega_{2}-\omega_{0}\right) t}
$$

represents the complex incident wave at the input port of the power amplifier. Thus, the two-tone excitation test can be seen as a modulated signal, both in amplitude and phase, having complex modulation envelope $\bar{a}(t)$.

The instantaneous reflected wave at the output port of the power amplifier can be analogously written as:

$$
b_{\text {out }}(t)=\operatorname{Re}\left\{\bar{b}(t) \cdot e^{j \omega_{0} t}\right\}
$$

where $\bar{b}(t)$ is the complex reflected wave at the output port of the power amplifier. Since $\Delta \omega<<\omega_{0}$, the complex quantities $\bar{a}(t)$ and $\bar{b}(t)$ are only slowly time-varying. 
For this reason, a quasi-stationary (memoryless) description of the power amplifier can be adopted:

$$
\bar{b}(t)=\bar{H}\left\{\omega_{0},|\bar{a}(t)|\right\} \cdot \bar{a}(t)
$$

where the $\bar{H}\{\}$ is a complex describing function depending on $\omega_{0}$ (frequency dependence in the small range $\omega_{0}-\Delta \omega / 2<\omega<\omega_{0}+\Delta \omega / 2$ is neglected) and on the absolute value of $\bar{a}(t)$ since time-invariance is implicitly assumed.

By considering the in-band signal components only (out-of-band signal components can be easily filtered out), the function $\bar{H}\{\cdot\}$ in (4) can be assumed to be even with respect to $|\bar{a}(t)|$, as it could be proved rigorously by means of Volterra analysis ${ }^{1}$ [3],[7]. Thus, we assume:

$$
\begin{gathered}
\bar{b}(t)=\bar{H}\left\{\omega_{0},|\bar{a}(t)|^{2}\right\} \cdot \bar{a}(t)= \\
=\left[H_{R}\left\{\omega_{0},|\bar{a}(t)|^{2}\right\}+j H_{I}\left\{\omega_{0},|\bar{a}(t)|^{2}\right\}\right] \cdot \bar{a}(t)
\end{gathered}
$$

where $H_{R}\{\cdot\}$ and $\left.H_{I}\{\}\right\}$ are the real and imaginary parts of $\bar{H}\{\cdot\}$.

By developing $H_{R}$ and $H_{I}$ in power series and omitting the explicit dependence on time, we obtain:

$$
\begin{aligned}
& \bar{b}=\left[\left(H_{R}^{S S}\left(\omega_{0}\right)+\alpha\left(\omega_{0}\right) \cdot|\bar{a}|^{2}+\gamma\left(\omega_{0}\right) \cdot|\bar{a}|^{4}+\ldots\right)+\right. \\
& \left.+j\left(H_{I}^{S S}\left(\omega_{0}\right)+\beta\left(\omega_{0}\right) \cdot|\bar{a}|^{2}+\delta\left(\omega_{0}\right) \cdot|\bar{a}|^{4}+\ldots\right)\right] \cdot \bar{a}
\end{aligned}
$$

where $H_{R}^{S S}, H_{I}^{S S}$ are the real and imaginary parts of the small-signal component of $\bar{H}\{$. $\}$ (i.e., $\bar{H}^{S S}\left(\omega_{0}\right)=$ $\left.\bar{H}\left\{\omega_{0}, 0\right\}\right)$, and $\alpha, \beta, \gamma, \delta, \ldots$ are suitable polynomial coefficients to be determined.

By substituting the complex incident wave (2) into (6) the reflected wave at the PA output port can be written after simple algebraic manipulation as:

$$
\begin{gathered}
\bar{b}=\bar{A} \cdot e^{j\left(\omega_{1}-\omega_{0}\right) \cdot t}+\bar{B} \cdot e^{j \cdot\left(\omega_{2}-\omega_{0}\right) \cdot t}+ \\
+\bar{C} \cdot e^{j \cdot\left(2 \omega_{1}-\omega_{2}-\omega_{0}\right) \cdot t}+\bar{D} \cdot e^{j \cdot\left(2 \omega_{2}-\omega_{1}-\omega_{0}\right) \cdot t}+\ldots
\end{gathered}
$$

where $\bar{A}, \bar{B}, \bar{C}, \bar{D}$ are complex coefficient depending on $H_{R}^{S S}, H_{I}^{S S}$, on the polynomial coefficients $\alpha, \beta$, $\gamma, \delta, \ldots$ and on the input levels $\bar{a}_{1}, \bar{a}_{2}$. In particular, in the simplest case of a second-order series expansion, we obtain:

$$
\begin{aligned}
& \bar{A}=\bar{a}_{1} \cdot\left[H_{R}{ }^{S S}+j H_{I}{ }^{S S}+(\alpha+j \beta) \cdot\left(2\left|\bar{a}_{2}\right|^{2}+\left|\bar{a}_{1}\right|^{2}\right)\right] \\
& \bar{B}=\bar{a}_{2} \cdot\left[H_{R}{ }^{S S}+j H_{I}{ }^{S S}+(\alpha+j \beta) \cdot\left(2\left|\bar{a}_{1}\right|^{2}+\left|\bar{a}_{2}\right|^{2}\right)\right] \\
& \bar{C}=\bar{a}_{1}^{2} \cdot \bar{a}_{2}^{*} \cdot(\alpha+j \beta)=\left|\bar{a}_{1}\right|^{2} \cdot\left|\bar{a}_{2}\right| \cdot e^{j \cdot\left(2 \angle \bar{a}_{1}-\angle \bar{a}_{2}\right)}(\alpha+j \beta) \\
& \bar{D}=\bar{a}_{2}^{2} \cdot \bar{a}_{1}^{*} \cdot(\alpha+j \beta)=\left|\bar{a}_{1}\right| \cdot\left|\bar{a}_{2}\right|^{2} \cdot e^{j \cdot\left(2 \angle \bar{a}_{2}-\angle \bar{a}_{1}\right)}(\alpha+j \beta)
\end{aligned}
$$

\footnotetext{
${ }^{1}$ In the case of not-even dependence in (4), no small signal solution would be definable. Thus, dependence of $\bar{H}$ on $|\bar{a}(t)|^{2}$ is considered in (5) instead on $|\bar{a}(t)|$ as in (4).
}

By considering two tone having the same amplitude, i.e., $\left|\bar{a}_{1}\right|=\left|\bar{a}_{2}\right|=\hat{a}$, and assuming: $\bar{a}_{1}=\hat{a}$ (zero phase, without loss of generality); $\bar{a}_{2}=\hat{a} \cdot e^{j<\bar{a}_{2}}$, we obtain:

$$
\begin{gathered}
|\bar{A}|=|\bar{B}|=\hat{a} \cdot\left|H_{R}^{S S}+j H_{I}^{S S}+3(\alpha+j \beta) \cdot \hat{a}^{2}\right| \cong \\
\cong \hat{a} \cdot\left|H_{R}^{S S}+j H_{I}^{S S}\right| \\
|\bar{C}|=|\bar{D}|=\hat{a}^{3} \cdot|\alpha+j \beta|
\end{gathered}
$$

where the approximation in (12) holds for sufficiently low signal levels such as those involved in IM distortion tests.

From (12) and (13) it is straightforward to derive the power of the spectral components at the output of the power amplifier. In particular:

$$
\begin{gathered}
P_{1}=\frac{1}{2} \cdot|\bar{A}|^{2}=\frac{1}{2} \hat{a}^{2} \cdot\left[\left(H_{R}{ }^{S S}\right)^{2}+\left(H_{I}^{S S}\right)^{2}\right] \\
P_{3}=\frac{1}{2} \cdot|\bar{C}|^{2}=\frac{1}{2} \hat{a}^{6} \cdot\left(\alpha^{2}+\beta^{2}\right)
\end{gathered}
$$

represent the power associated with the fundamental and the third-order IM products.

Third-order intercept point $\left(\mathrm{IP}_{3}\right)$ can be then easily evaluated by means of well-known formulas:

$$
\begin{gathered}
I P_{3}=\frac{3 \cdot P_{I}^{d B m}-P_{3}^{d B m}}{2}= \\
=30 \log \left|H_{R}^{S S}+j H_{I}^{S S}\right|-10 \log |\alpha+j \beta|+10 \log (0.5)
\end{gathered}
$$

Equations (14)-(16) allow for the prediction of IMD in power amplifiers provided that the two scalar coefficient $\alpha, \beta$, are identified. To this aim, a single-tone Harmonic Balance simulation can be carried out, provided that a suitably accurate non-linear dynamic model of the power amplifier (i.e., of the electron devices) is available. Simple analytical mean square minimization of discrepancies between (6) and single-tone HB-simulated results provides simple and reliable identification of the two coefficients.

Obviously, by taking into account higher-order terms in the polynomial expansion (6), higher order IM products can also be evaluated. For instance, by taking into account fourth-order terms in (6) the fifth-order IM products (and fifth order intercept point $\mathrm{IP}_{5}$ ) can be predicted.

\section{EQUIVALENT CONSTRAINTS ON THE MAXIMUM ACCEPTABLE INTERMODULATION DISTORTION}

We consider the following quantity, corresponding to an amplifier complex-gain compression [9]:

$$
G\left(|\bar{a}|^{2}\right)=\left|\frac{\bar{H}\left\{|\bar{a}|^{2}\right\}-\bar{H}^{S S}}{\bar{H}^{S S}}\right|
$$

where $\bar{H}\{\}$ is the complex describing function given in (5) and $\bar{H}^{S S}$ is its small signal value (dependence on $\omega_{0}$ omitted). The $G\left(|\bar{a}|^{2}\right)$ parameter defined in (17) can be related to conventional IMD evaluation indexes with 
the important advantage of transforming a power amplifier design specification on maximum (two-tone excitation) intermodulation distortion into an equivalent specification on a (single-tone excitation) complex-gain compression (both $\mathrm{AM} / \mathrm{AM}$ and $\mathrm{AM} / \mathrm{PM}$ characteristics involved). In particular, simple substitution of the polynomial expansion of $\bar{H}($.$) , as suggested by (6), into$ (17) leads to:

$G\left(|\bar{a}|^{2}\right)=\frac{\left.|(\alpha+j \beta)| \bar{a}\right|^{2}+(\gamma+j \delta)|\bar{a}|^{4}+\ldots \mid}{\left|\bar{H}^{S S}\right|} \cong \frac{|\alpha+j \beta|}{\left|\bar{H}^{S S}\right|} \cdot|\bar{a}|^{2}$

When $\bar{a}$ represents the complex envelope associated with a two-tones input test signal at frequencies $\omega_{1}, \omega_{2}$ sharing the same amplitude $\hat{a}$, (18) becomes slowly timevarying since (after simple algebraic manipulation):

$$
|\bar{a}(t)|^{2}=2 \hat{a}^{2}\left(1+\cos \left[\left(\omega_{1}-\omega_{2}\right) t+\angle \bar{a}_{1}-\angle \bar{a}_{2}\right]\right) .
$$

Thus, (18) ranges between 0 and a peak value obtained when $|\bar{a}|^{2}=4 \hat{a}^{2}$. Moreover, by using (14)-(15), the $\mathrm{IMR}_{3}$ figure of merit can be written as:

$$
I M R_{3} \doteq \frac{P_{3}}{P_{1}} \cong \frac{|\alpha+j \beta|^{2}}{|\bar{H} S S|^{2}} \cdot \hat{a}^{4} .
$$

We define now a non-linearity index $\varepsilon$ as the peak value of $G\left(|\bar{a}|^{2}\right)$. Thus, by considering (18),(19),(20) the maximum intermodulation distortion design specification: $I M R_{3}<\delta$, is equivalently satisfied by the following

\section{$\operatorname{Real}\{\bar{H}\}$}

$\alpha$ Identification

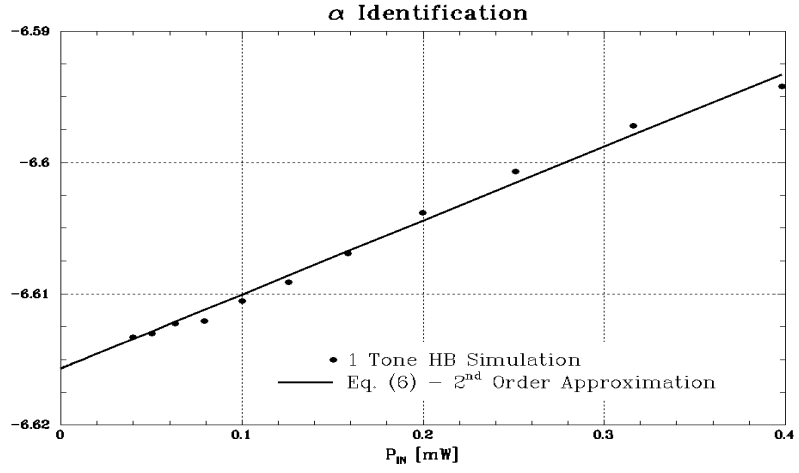

constraint on the non-linearity index $\varepsilon$ ( $\bar{H}$ evaluation in the two-tones input case):

$$
\varepsilon \doteq \frac{|\alpha+j \beta|}{\left|\bar{H}^{S S}\right|} \cdot 4 \hat{a}^{2}=4 \sqrt{I M R_{3}}<4 \sqrt{\delta} .
$$

When considering, instead, the case of a single-tone input test signal at frequency $\omega_{0}$ with amplitude $\hat{a}$, the complex-gain compression $G\left(|\bar{a}|^{2}\right)$ keeps constant with time. However, it can still be related to the $I M R$ of an "associated" intermodulation test where the two-tones have both amplitude equal to $\hat{a}$. In this case, the design constraint $I M R_{3}<\delta$ is found equivalent to ( $\bar{H}$ evaluation in the single-tone input case):

$$
\varepsilon \doteq \frac{|\alpha+j \beta|}{|\bar{H} S S|} \cdot \hat{a}^{2}=\sqrt{I M R_{3}}<\sqrt{\delta}
$$

According to (22), $I M R_{3}$ values corresponding to some non-linearity index $\varepsilon$ levels are shown in Table I.

\begin{tabular}{|c|c|}
\hline$\varepsilon[\%]$ & $I M R_{3}[\mathrm{dBc}]$ \\
\hline 5 & -26 \\
\hline 3 & -30.5 \\
\hline 1 & -40 \\
\hline .5 & -46 \\
\hline
\end{tabular}

TABLE I

RELATIONSHIP BETWEEN NON-LINEARITY INDEX $\varepsilon$ AND INTERMODULATION DISTORTION

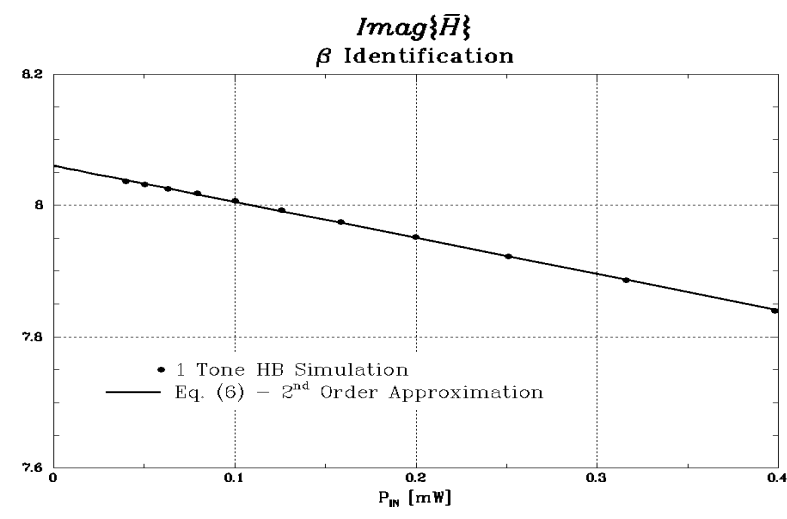

Figure 1. Complex transfer function $\bar{H}\{0$ (real and imaginary part) versus input available power.

Prediction by HB-analysis (dot) and by means of (6) (line) - II-order approximation case.

$\operatorname{Real}\{\bar{H}\}$

$\alpha$ and $\gamma$ Identification

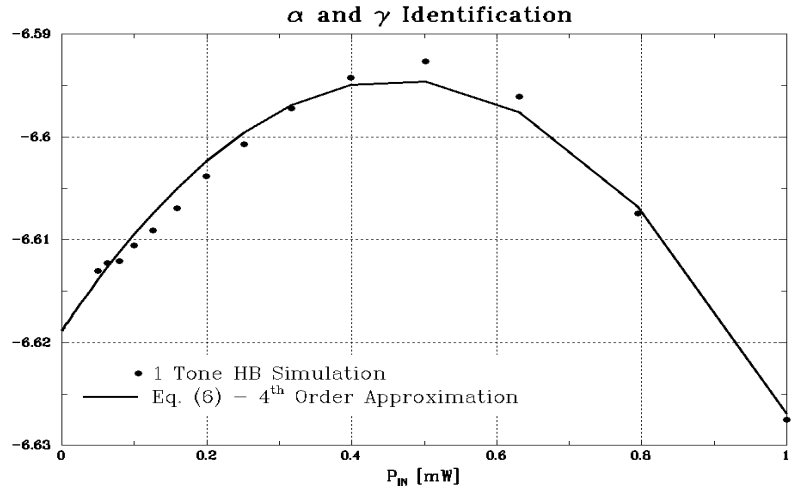

$\operatorname{Imag}\{\bar{H}\}$

$\beta$ and $\delta$ Identification

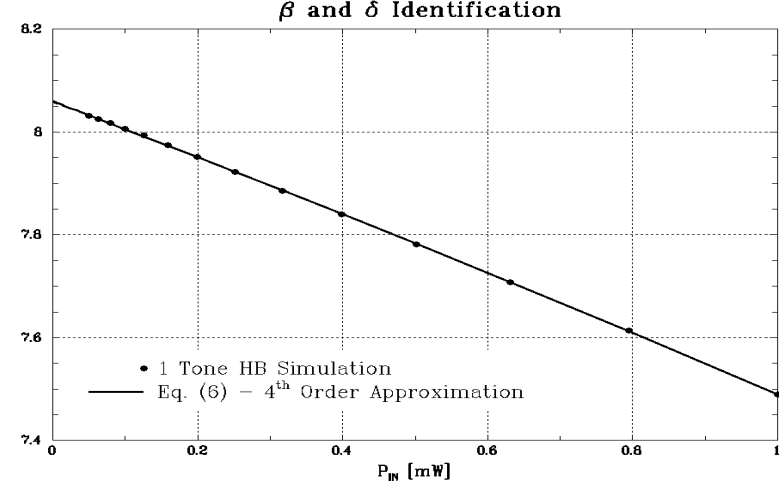

Figure 2. Complex transfer function $\bar{H}\{\}$ (real and imaginary part) versus input available power. Prediction by HB-analysis (dot) and by means of (6) (line) - IV-order approximation case. 


\section{EXPERIMENTAL VALIDATION}

In order to test the IM distortion prediction accuracy of

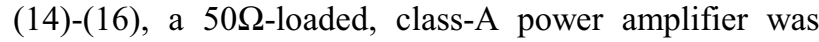
considered, based on a $600 \mu \mathrm{m}$ GaAs PHEMT. An accurate dynamic non-linear model of the electron device was identified, namely the Non-linear Discrete Convolution (NDC) model [10], by choosing a suitable memory time $\left(\mathrm{T}_{\mathrm{M}}\right)$ equal to $2 \mathrm{ps}$.

In order to identify the $\alpha, \beta, \gamma, \delta, \ldots$ polynomial coefficients, a single-tone power-swept Harmonic Balance simulation was then carried out, and the analytical least-square best-fit problem was solved for the minimum discrepancies between (6) and the single-tone HB results. Two cases have been considered here: second-order and fourth-order polynomial expansion in (6). Fig. 1. shows the complex transfer function $\bar{H}\{\cdot\}$ (real and imaginary part) versus input available power $\left(1 / 2 \cdot \hat{a}^{2}\right)$ predicted by HB analysis and by means of (6) in the second-order expansion case, after the best-fit solution for $\alpha, \beta$. Input power levels not higher than -4 $\mathrm{dBm}$ are here considered for polynomial fitting in order to avoid ranges where the $\bar{H}\{\}$ behavior depends on fourth and higher order powers of $|\bar{a}|$. Instead, Fig. 2. shows analogous results in the case of fourth-order expansion of (6). Higher input power levels are considered here, up to $0 \mathrm{dBm}$ in order to get better identification of the four $\alpha, \beta, \gamma$, and $\delta$ coefficients.

\begin{tabular}{|c|c|c|c|}
\cline { 2 - 4 } \multicolumn{1}{c|}{} & \multicolumn{2}{c|}{ IP3 [dBm] } & IP5 [dBm] \\
\hline \multirow{2}{*}{$\begin{array}{c}\text { Proposed } \\
\text { procedure }\end{array}$} & $\begin{array}{c}\text { II-order } \\
\text { in (6) }\end{array}$ & $\begin{array}{c}\text { IV-order } \\
\text { in (6) }\end{array}$ & $\begin{array}{c}\text { IV-order } \\
\text { in (6) }\end{array}$ \\
\cline { 2 - 4 } Two-tone HB & 33.12 & 33.18 & 30.09 \\
\hline \multicolumn{2}{|c|}{31.71} & 29.62 \\
\hline
\end{tabular}

TABLE II

Third- and fifth-order intercept point. Prediction by means of (16) and by means of two-tone HB analysis.

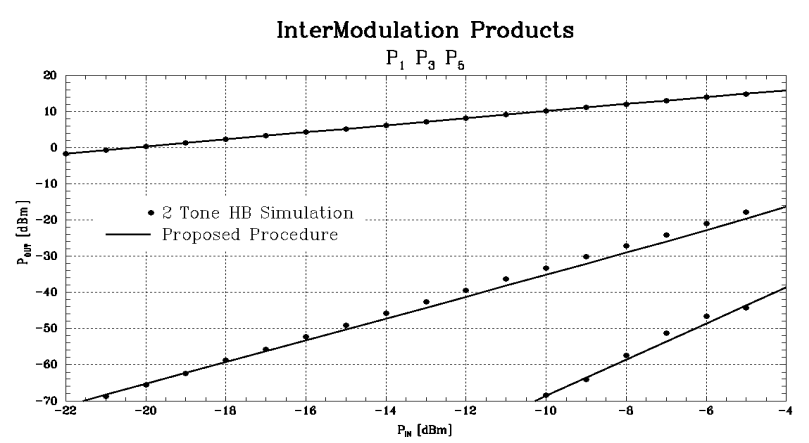

Figure 3. Fundamental, third- and fifth-order IM products vs. input SCL available power. Comparison between prediction by means of (6) with IV-order approximation and two-tone HB analysis.

By using the extraction results, third-order IM distortion was predicted by means of (14)-(16). Fifthorder IM prediction was also possible in the second case considered. Predicted IMD results were finally compared with much more time-consuming two-tone HB simulation of the same amplifier. Comparisons are provided in Table II. As expected, prediction results are in good agreement, especially in the more accurate case where fourth-order expansion of (6) was taken into account. Thus, the simplified, computationally-efficient approach presented here allows for accurate description of the mild distortion in quasi-linear power amplifiers by means of single-tone Harmonic Balance simulations, strongly shortening the PA performance evaluation time, especially important in iterative design procedures.

\section{CONCLUSION}

The paper has shown how intermodulation distortion in highly linear power amplifiers for microwave communication can be accurately predicted on the basis of a single-tone power-swept Harmonic Balance analysis, without need for time-consuming and computationally expensive two-tone analyses. Validation of the method

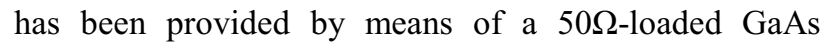
$600 \mu \mathrm{m}$-PHEMT based power amplifier simulated with Agilent ADS.

Moreover a non-linearity index $\varepsilon$, involving both $\mathrm{AM} / \mathrm{AM}$ and AM/PM amplifier characteristics, has been introduced and equivalence conditions between specifications on maximum acceptable IMD and maximum $\varepsilon$ have been found. On the theoretical basis presented in this paper, numerically efficient procedures for the design of highly linear power amplifiers are actually under development.

\section{REFERENCES}

[1] R. S. Carson, Radio Communications Concepts: Analog, John Wiley \& Sons, 1990.

[2] S. A. Maas, Nonlinear Microwave Circuits, Artech House, 1988.

[3] S. A. Maas, "Volterra analysis of spectral regrowth", IEEE Microwave and Guided Wave Letters, vol 7, no. 7, Jul 1997.

[4] J. S. Kenney, A. Leke, "Power amplifier spectral regrowth for digital cellular and PCS applications", Microwave Journal, Oct 1995 pp. 74-92.

[5] H. Gutierrez, K. Gard, M. B. Steer, "Nonlinear gain compression in microwave amplifiers using generalized power-series analysis and transformation of input statistics", IEEE Trans. on MTT, vol 48, no. 10, Oct 2000.

[6] J. C. Pedro, N. B. de Carvalho, "On the use of multitone techniques for assessing RF components' intermodulation distortion", IEEE Trans. on MTT, vol 47, no. 12, Dec 1999.

[7] T. Wang, T. J. Brazil, “The estimation of volterra transfer functions with applications to RF power amplifier behavior evaluation for CDMA digital communication", IEEE MTT$S$ Digest, June 2000.

[8] G. T. Zhou, J. S. Kenney, "Predicting spectral regrowth of nonlinear power amplifiers", IEEE Trans. on Communications, vol 50, no. 5, May 2002.

[9] R. P. Paganelli, A. Santarelli, G. Zucchelli, A. Costantini, G. Vannini, F. Filicori, "A computationally efficient approach for the design of RF power", in Proc of the GAAS2002, Milan, Italy, Sep 2002, pp ${ }^{\circ} 405-408$.

[10] F. Filicori, A. Santarelli, P. Traverso, G. Vannini, "Electron device model based on nonlinear discrete convolution for large-signal circuit analysis using commercial CAD packages", in Proc. of the GAAS99, Munich, Germany, Oct 1999. 